\title{
Concentration fluctuations in polymer gel investigated by neutron scattering: Static inhomogeneity in swollen gel
}

\author{
Satoshi Koizumia) \\ ASRC, Japan Atomic Energy Research Institute, Tokai, Ibaraki-ken 319-1195, Japan \\ Michael Monkenbusch, Dieter Richter, and Dietmar Schwahn \\ IFF, Forschungszentrum Jülich GmbH, D52425 Jülich, Germany \\ Bela Farago \\ Institute of Laue-Langevin, 38042 Grenoble, France
}

(Received 18 February 2004; accepted 30 September 2004)

\begin{abstract}
By using small-angle neutron scattering (SANS) and neutron spin echo (NSE), we have quantitatively investigated the static inhomogeneity in poly (N-isopropyl acrylamide) gel (PNIPA) in microscopic length scales of $0.015<q<0.16 \mathrm{~A}^{-1}$, where $q$ is a wave number of scattered neutrons. NSE revealed that at lower $q\left(\cong 0.015 \mathrm{~A}^{-1}\right)$, the concentration fluctuations in the PNIPA gel decays more slowly as compared to the PNIPA solution without crosslinks. According to our scenario that the slower decay found for the PNIPA gel is due to the static inhomogeneity coexisting in the swollen gel, small-angle scattering $S(q)$ obtained by SANS has been quantitatively decomposed into thermal and static scattering components, respectively, $S_{\mathrm{th}}(q)$ and $S_{\mathrm{st}}(q)$. It was further revealed that (i) the $q$-region where $S_{\text {st }}(q)$ becomes dominant is closely related to that for the abnormal butterfly scattering under stretching, and (ii) as the temperature increases toward the temperature for volume phase transition, $S_{\mathrm{st}}(q)$ of a squared Lorentzian shape increases more drastically than $S_{\mathrm{th}}(q)$ of a Lorentzian shape. These findings were quantitatively understood in the theoretical framework by Panyukov and Rabin [Macromolecules 29, 7960 (1996)] or by Onuki [J. Phys. II. France 2, 45 (1992)], taking into account stress-fluctuation coupling under coexistence of the inherent structural heterogeneity in the real gel. We further found that the static inhomogeneity showing $S_{\mathrm{st}}(q)$ seems to relate to the necklacelike microstructure, appearing after a shallow quench into the collapsed phase. (c) 2004 American Institute of Physics. [DOI: 10.1063/1.1823411]
\end{abstract}

\section{INTRODUCTION}

In this paper, we investigate static inhomogeneity in the real polymer gel, focusing on microscopic length scales where the gel changes from a continuous media to a molecular structure. By employing neutron scattering, we aim to quantitatively determine the static inhomogeneity, which appears upon swelling or deformation as a result of stressfluctuation coupling ${ }^{1}$ under coexistence of the structural heterogeneity in the real gel. The polymer gels are composed of a networked structure of giant molecules in suspension, which are associated by a variety of interactions of covalent bond (chemical gel) or hydrogen bond, hydrophobic interaction, van der Waals interaction and topological constraint (physical gel). The characteristic times of these interactions determine the rheological relaxation of gels. The slower component of the networked structure is immersed in the matrix of faster component of solvents, which we call $d y$ namically asymmetric. When the polymer gel is subject to swelling or deformation, the gel changes the network structure in order to minimize free energy due to locally imbalanced stress associated by the network. Thus the stress and

\footnotetext{
a) Author to whom correspondence author should be addressed. Telephone: +81-29-284-3511. Fax: +81-29-282-5939. Electronic mail: koizumi@neutrons.tokai.jaeri.go.jp
}

concentration fluctuations are strongly coupled in the gel (stress-fluctuation coupling). ${ }^{1}$ Due to the coupling effect, the heterogeneous crosslink density in the real gel induces the excess concentration fluctuations (Static Inhomogeneity). ${ }^{2}$ Here the notation of "static" means "nondecaying" as the time proceeds.

The stress-fluctuation coupling effect has been intensively studied during the past decade especially from a dynamic point of view. ${ }^{3}$ At first, we overview it with respect to dynamical symmetry or asymmetry. For dynamically symmetric mixtures, whereby two constituents are similar in mobility, the local stress associated with the individual constituents is balanced during the flow of cooperative diffusion or phase separation. In this case, the stress-fluctuation coupling effect does not occur and the dynamical universality is held even for viscoelastic materials as well as for simple liquids. The universal behaviors are reported as follows. The first example is that in the late stage of spinodal decomposition, the scaling law of $R / \sim(t / \tau)^{\alpha}$ well describes the coarsening late-stage dynamics, where $R$ is a characteristic size of droplets, $t$ is the phase separation time and $\alpha$ is the growth exponent. ${ }^{3} \xi$ and $\tau$ are the correlation length and characteristic lifetime of fluctuations. This finding means that all microscopic details are not relevant to determine the selfsimilar nature of domain growth during the phase separation. Another example is that the cooperative diffusion is simply 
controlled only by thermodynamics of the mixture. The cooperative diffusion coefficient $D_{c}$ is given by $D_{c}$ $=\Lambda(q) q^{2} S(q)^{-1}$ where $\Lambda(q)$ and $S(q)$ the Onsager kinetic coefficient (or self-diffusion constant) and the static structure factor, respectively. ${ }^{4}$

The dynamical asymmetry, on the other hand, causes the stress-fluctuation coupling effect and strongly affects thermal concentration fluctuations during cooperative diffusion or phase separation. Note that the dynamical asymmetry is the case not only for the gels but also for the sols. During the flow, the local stress, which is imbalanced between faster and slower moving components, affects the diffusional process. Due to the dynamical coupling effect, we can observe a variety of unusual phenomena, as follows. The real space observation by light microscope revealed the unusual phase separation named as "viscoelastic phase separation" 3 and a variety of unusual patterns, such as a moving droplet, a networklike domain and phase inversion. ${ }^{3}$ This is because during the flow of phase separation, the slow component cannot catch up with deformation caused by the phase separation and starts to behave like an elastic body. In a single phase of polymer solutions, a nonexponential relaxation of concentration fluctuations is observed by dynamic light scattering. ${ }^{5}$ SANS revealed that in the polymer mixture with large difference in glass transition temperature $T g$, concentration fluctuations are suppressed by the imbalanced stress field when the mixture stays in a gel-like limit. ${ }^{6}$

The permanent crosslinks in the chemical gel prohibit polymer chains to change relative positions. Therefore, the concentration fluctuation in the gel is strongly coupled with the stress associated by the gel network. The permanent crosslink induces the elastic moduli and affects not only the flow at a nonequilibrium state but also free energy of the gel. Therefore, due to the elastic property, three instabilities are reported for the polymer gel; ${ }^{7}$ macroscopic instability of volume phase transition for $K_{\mathrm{os}}^{\mathrm{gel}}<0$ at $T=T_{V}$, surface instability for $K_{\mathrm{os}}^{\mathrm{gel}}+\left(\frac{1}{3}\right) \mu<0$ and bulk instability of spinodal decomposition for $K_{\mathrm{os}}^{\mathrm{gel}}+\left(\frac{4}{3}\right) \mu<0$ at $T=T_{s}$ where $K_{\mathrm{os}}^{\mathrm{gel}}$ and $\mu$ are the osmotic bulk and shear moduli for the gel, respectively. Above $T_{V}$, the gel shrinks and its dynamics is governed by a cooperative diffusion process (Diffusion Limited Model $)^{8}$ where the cooperative diffusion coefficient $D_{c}$ is given as $D_{c}=\left[K_{\mathrm{os}}^{\mathrm{gel}}+\left(\frac{4}{3}\right) \mu\right] / f$ where $f$ is a friction coefficient. Simultaneously during squeezing, the shear modulus $\mu$ plays an important role to keep a shape of the gel.

Viscoelastic phase separation occurs in the gel when $K_{o s}+4 \mu / 3<0 .{ }^{9}$ Due to the stress-fluctuation coupling in the gel, the networklike ${ }^{10}$ or spongelike ${ }^{11}$ microdomain appears and its domain growth is pinned by the networked structure in the gel. The microdomain in the collapsed phase has been studied by SANS $;{ }^{12}$ the necklacelike or networklike domain appears in a solvent-rich matrix, respectively, for a shallow or deep quench above $T_{V}$. It should be noted that the chemically crosslinked network structure is crucial to pin the domain growth at the length scales of the gel network and its inherent heterogeneity.

Now we consider the real gel, having the heterogeneous microstructures such as loops, dangling chain ends and het-

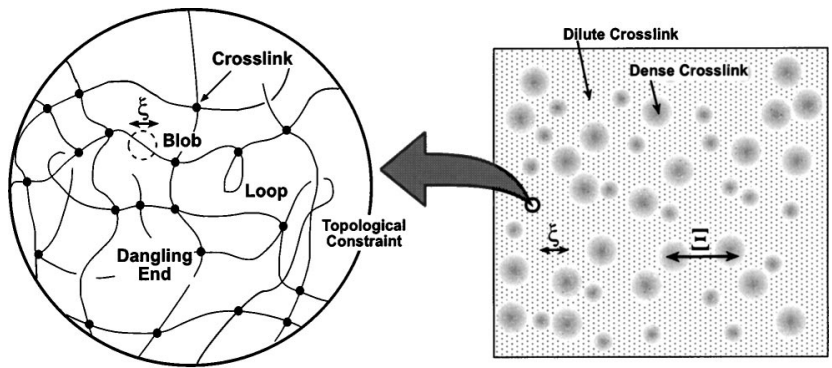

(a) Molecular Structure Limit

(b) Continuous Media Limit

FIG. 1. Schematic diagram for inherent heterogeneities in the real gel; (a) microscopic heterogeneities of dangling chain ends and loops in the molecular structure limit and (b) heterogeneous crosslink density with a characteristic length scale $\Xi$ in the continuous media limit.

erogeneous density of crosslinks, which are generally introduced during a gelation process (see Fig. 1). The static inhomogeneity is also influenced by such heterogeneous microstructures. The static inhomogeneity is more enhanced when the gel is subject to swelling by changing solvent quality, temperature, or pressure. This is because the permanent crosslinks restrict reconfiguration of the network structure so that the poorly crosslinked region is more swollen, whereas the densely crosslinked region is less swollen. Due to the static inhomogeneity, the real gel shows so-called overscattering in a small-angle scattering region as compared to the polymer solution. ${ }^{2}$ When the gel is uniaxially deformed, the overscattering is further developed as a so-called abnormal butterfly pattern, i.e., enhancement of scattering intensity along a deformation direction. ${ }^{2}$

The static inhomogeneity in a polymer gel has been investigated by light scattering. Note that since the wavelength of light scattering is order of $0.1 \mu \mathrm{m}$, the light scattering studies the static inhomogeneity in a continuum limit. Static light scattering with a narrow beam scanning in the gel detected the speckle scattering pattern due to the static inhomogeneity. ${ }^{13}$ The speckles appear on a positioninsensitive base scattering, which is attributed to the thermal concentration fluctuations. ${ }^{13}$ The ratio of scattering intensity between the averaged speckle and the base scattering agrees with the nondecaying plateau level determined by dynamic light scattering. ${ }^{13}$ The fraction of the static component depends on the gelation conditions. ${ }^{14}$ The speckle was discussed as related to the abnormal butterfly pattern. ${ }^{15}$

In this paper, we microscopically investigate the static inhomogeneity in a hydrogel of a poly(N-isopropyl acrylamide) (PNIPA). As using a notation of "microscopic," we focus on length scales where the gel changes from a continuum to a molecular structure (Fig. 1). For this purpose, we employed SANS and neutron spin echo (NSE). A static scattering function $S(q)$ obtained by SANS is a snap shot of the concentration fluctuations, where $q$ is an amplitude of scattering vector $[q=(4 \pi / \lambda) \sin (\theta / 2)$ and $\lambda$ or $\theta$ is wavelength or scattering angle, respectively]. NSE, on the other hand, provides the normalized intermediate scattering function of $S(q, t) / S(q)$ within a time domain up to order of $100 \mathrm{~ns}{ }^{16}$ By combining SANS and NSE, we are able to obtain a timeevolving picture of the concentration fluctuations in reciprocal space. With these scattering methods, we successfully 
decomposed $S(q)$ into two components of the thermal concentration fluctuations $S_{\mathrm{th}}(q)$ and the static inhomogeneity $S_{\mathrm{st}}(q)$. The abnormal butterfly scattering pattern under deformation will be discussed related to the static inhomogeneity thus determined. From temperature changes of $S_{\mathrm{th}}(q)$ and $S_{\mathrm{st}}(q)$, we discuss the macroscopic mechanical moduli, i.e., longitudinal elastic modulus $M\left[=K_{\mathrm{os}}^{\mathrm{gel}}+\left(\frac{4}{3}\right) \mu\right]$ and shear modulus $\mu$, or the Poisson ratio $\sigma$. The ratio of $M / \mu$ determined by neutron scattering varies from about 3 to 2 as the temperature increases from $20^{\circ} \mathrm{C}$ to $28^{\circ} \mathrm{C}$, which agrees with a change of the solvent quality from good to $\theta$-solvents. SANS further revealed that the static inhomogeneity grows up to the necklacelike microstructures above $T_{V}$. We discussed these experimental findings in comparison to the recent theoretical approaches developed by Rabin and Panyukov ${ }^{17}$ or by Onuki ${ }^{18}$ for the real gel having the inherent structural heterogeneity.

\section{EXPERIMENTS}

\section{A. Sample specimen}

We studied a hydrogel of PNIPA, crosslinked with 0.32 wt $\%$ of $\mathrm{N}, \mathrm{N}$-methylenebisacrylamide (BIS). A N-isopropylacrylamide (NIPA) monomer (Kohjin Co. Ltd., Fuji, Japan) was first purified by re-crystallizing in its toluene solution at $4{ }^{\circ} \mathrm{C}$. After washing and filtering the re-crystallized NIPA monomer in cold hexane, the NIPA monomer was further dried in vacuum at $25^{\circ} \mathrm{C}$. The pre-gel solution was prepared by dissolving the NIPA monomer of $7.8 \mathrm{~g}, \mathrm{~N}$, $\mathrm{N}$-methylenebisacrylamide (BIS) of $0.345 \mathrm{~g}$, and ammoniumpersulfate of $0.043 \mathrm{~g}$ into $\mathrm{D}_{2} \mathrm{O}$ of $100 \mathrm{ml}$. After complete dissolution, the pre-gel solution was degassed and then kept in a refrigerator for $30 \mathrm{~min}$ so as to avoid gelation. Then an initiator, $\mathrm{N}, \mathrm{N}, \mathrm{N}^{\prime}, \mathrm{N}^{\prime}$-tetramethylethylenediamine of $240 \mu \mathrm{l}$ was added to the pre-gel solution to initiate polymerization and crosslinking. During polymerization, the pre-gel solution was poured into a glass plate mold having gaps of $1.5 \mathrm{~mm}$ thickness and was gelled at $10^{\circ} \mathrm{C}$ for $24 \mathrm{~h}$ (reference state). The gelation temperature of $10.0^{\circ} \mathrm{C}$, which is far from the cloud point of the PNIPA solution $T_{C}\left(=33.0^{\circ} \mathrm{C}\right)$, is crucial in order to obtain a transparent gel. The PNIPA gel thus prepared contains water of $93.0 \mathrm{wt} \%$ at the reference temperature of $10^{\circ} \mathrm{C}$. The prepared gels were washed with an excess amount of $\mathrm{D}_{2} \mathrm{O}$. We also synthesized un-crosslinked PNIPA polymers with the same receipt described here, except for BIS. For SANS measurements, we prepare a polymer solution of un-crosslinked NIPA of $7.0 \mathrm{wt} \%$ in $\mathrm{D}_{2} \mathrm{O}$.

The infinite connectivity of partial chains is crucial to zoom in a microscopic coil-globule transition up to macroscopic length scales (volume phase transition). ${ }^{19}$ For the NIPA gel in $\mathrm{D}_{2} \mathrm{O}$, the volume phase transition occurs at $T_{V}$ $=33.5^{\circ} \mathrm{C}$; below $T_{V}$, the NIPA gel swollen with water (swollen phase), while above $T_{V}$, it shrinks (collapsed phase). Note that $T_{V}$ for the NIPA gel is $0.5^{\circ} \mathrm{C}$ higher than $T_{C}$ for the NIPA solution.

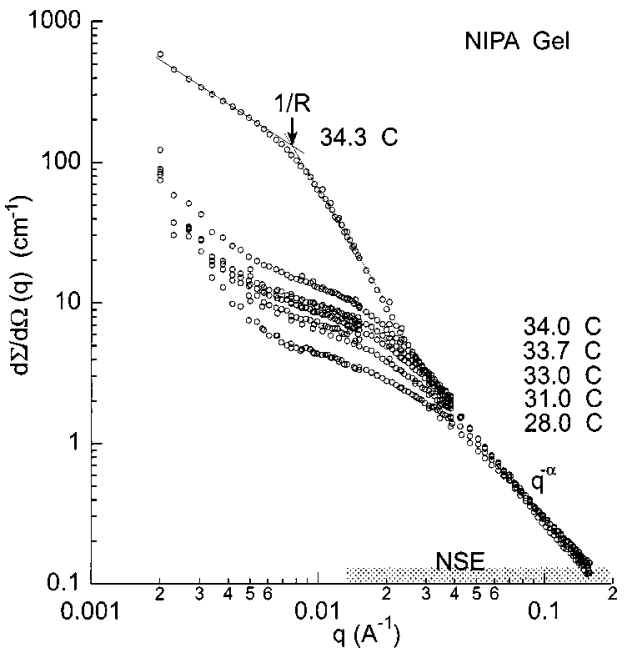

FIG. 2. Scattering $q$-profiles obtained by SANS for NIPA gel near volume phase transition temperature $T_{V}$.

\section{B. Neutron scattering}

SANS measurements were performed with KWS1 at the Forschungszentrum Jülich (IFF) at Jülich in Germany and with SANS-J at Japan Atomic Energy Research Institute (JAERI) at Tokai in Japan. In SANS experiments, the scattered neutrons are collected by an area detector which covers a wide $q$-range of $0.002<q<0.2 \mathrm{~A}^{-1}$, by changing detector positions. The two-dimensional data set thus obtained was corrected for counting efficiency, instrumental background and air scattering on a pixel-to-pixel basis. After circularaveraging, we converted scattering intensity to the absolute differential cross section per a sample volume $[d \Sigma / d \Omega(q)$ in unit of $\left.\mathrm{cm}^{-1}\right]$, by using a pre calibrated secondary standard of a Lupolen film. ${ }^{20}$ or irradiated Aluminum. Finally, the incoherent scattering of hydrogen was subtracted as a background by using the higher $q$-region where the scattering profile becomes $q$-independent. The gel was immersed in a sample holder with a large amount of $\mathrm{D}_{2} \mathrm{O}$, which was temperature-controlled in a heater furnace for SANS. Under isothermal conditions, the gel in a holder satisfies an equilibrium condition, changing its volume and, therefore, its water content. The sample thickness of $1.5 \mathrm{~mm}$ thickness gave transmissions ranging from 0.8 to 0.65 .

Neutron spin echo (NSE) measurements were performed with FRJ2-NSE ${ }^{21}$ in the Forschungszentrum Jülich in Germany and with $\mathrm{IN} 15^{22}$ in the institute of Laue-Langevin in France. The sample temperatures were changed from $20.0^{\circ} \mathrm{C}$ to $34.5^{\circ} \mathrm{C}$. FRJ2-NSE has a Fourier observation time $t_{F}$ up to $18 \mathrm{~ns}$ with an incident neutron of $\lambda=8 \mathrm{~A}$. IN15 has $t_{F}$ up to $190 \mathrm{~ns}$ with an incident neutron of $\lambda=15 \mathrm{~A}$. The decay curves were calibrated with an elastic scatterer of glassy carbon.

\section{EXPERIMENTAL RESULTS}

\section{A. Small-angle neutron scattering}

Figure 2 shows $q$-profiles $S(q)$ obtained by SANS both in the swollen and collapsed phases near $T_{V}$. As the temperature increases toward $T_{V}$, the scattering intensity in a 
low $q$-regime increases because of the lower critical solution temperature (LCST) phase diagram of the PNIPA-water mixture. ${ }^{23}$ In a high $q$-region, the small-angle scattering does not change showing an asymptotic $q$-behavior close to $q^{-2}$. This power law of $q^{-2}$ is attributed to a segmental distribution of PNIPA in a blob. Thus in the swollen phase, the $q$-profiles $S(q)$ are similar to that of semi dilute polymer solutions, showing the Ornstein-Zernike regime in low $q$ and the power law regime at high $q$. However, this first impression is not correct because the static inhomogeneity coexists with thermal concentration fluctuations. We aim to quantitatively decompose $S(q)$ into two scattering components of $S_{\mathrm{st}}(q)$ and $S_{\mathrm{th}}(q)$. For this purpose, we performed NSE measurements on both PNIPA gel and solution. The $q$-positions of NSE widely covers the Ornstein-Zernike and the power low regimes, as indicated in Fig. 2.

At $34.3^{\circ} \mathrm{C}$ in the collapsed phase, we observed strong increase of small-angle scattering and the $q$-behavior is different from the OZ type observed in the swollen phase. This increase of small-angle scattering is attributed to appearance of microstructures in the gel, i.e., the necklacelike microdomain rich in NIPA chains and dispersed in the water rich matrix. $^{12}$

Figure 3 shows two-dimensional scattering patterns of the PNIPA gel stretched with a deformation ratio $\Lambda=1.5$ at different temperatures of $28.0,31.0$, and $33.0^{\circ} \mathrm{C}$. Here we clearly recognize the abnormal butterfly scattering pattern where the iso-intensity lines are elongated along the deformation. After a sector-averaging over an azimuthal angle of $30^{\circ}$, we obtained $q$-profiles for two directions parallel and perpendicular to the deformation (see Fig. 4). For the parallel direction the enhancement of scattering intensity appears in a low $q$-region of $q<0.02 \mathrm{~A}^{-1}$, whereas for the perpendicular direction, the scattering intensity does not change as compared to the undeformed gel. The anisotropic enhancement of the scattering intensity in the $q$-region of $q<0.02 \mathrm{~A}^{-1}$ is further discussed related to $S_{\mathrm{st}}(q)$ determined by the NSE measurements.

\section{B. Neutron spin echo}

In the $q$-region indicated in Fig. 2, we performed NSE measurements by employing the two spectrometers IN15 at ILL and FRJ2-NSE at Jülich. Figure 5(a) shows the decay curves of NSE $S(q, t) / S(q, t=0)$ at three typical $q$-positions for both the NIPA gel and solution at $28.0^{\circ} \mathrm{C}$. The open and filled symbols indicate the decay curves for the PNIPA gel and solution, respectively. The large symbols covering shorter $t_{F}$ show the decay curves obtained by FRJ2-NSE, while the smaller symbols show those obtained by IN15. The shade shows the difference between PNIPA gel and solution at the same $q$-position.

We observed the significant retardation on decays for the NIPA gel in $0.035<q<0.1 \mathrm{~A}^{-1}$, as compared to the PNIPA solution. ${ }^{24}$ At $q=0.035 \mathrm{~A}^{-1}$, we can recognize the retardation of decay for the PNIPA gel for $t_{F}$ longer than $30 \mathrm{~ns}$, while for shorter $t_{F}$ of $22 \mathrm{~ns}$, two decay curves for the PNIPA gel and solution are identical. At $q=0.076 \mathrm{~A}^{-1}$, we recognize the retardation even at $t_{F}=22 \mathrm{~ns}$. As $q$ increases to the highest $q=0.16 \mathrm{~A}^{-1}$, the difference becomes negligibly (a) $28.0^{\circ} \mathrm{C}$

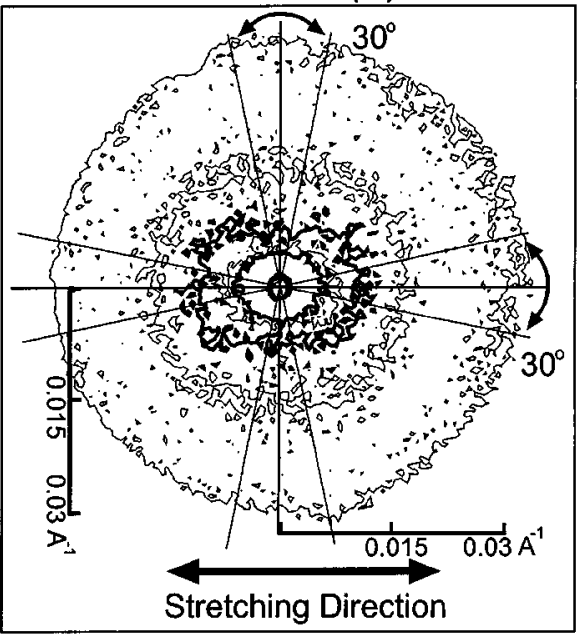

(b) $31.0^{\circ} \mathrm{C}$

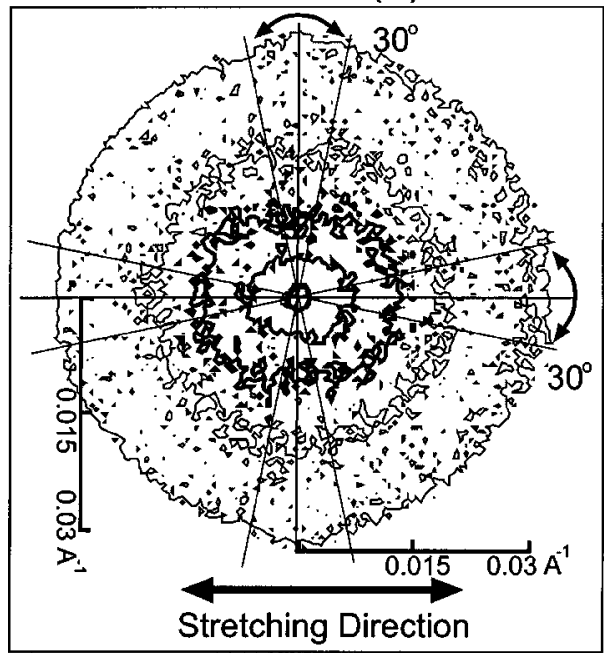

(c) $33.0^{\circ} \mathrm{C}$

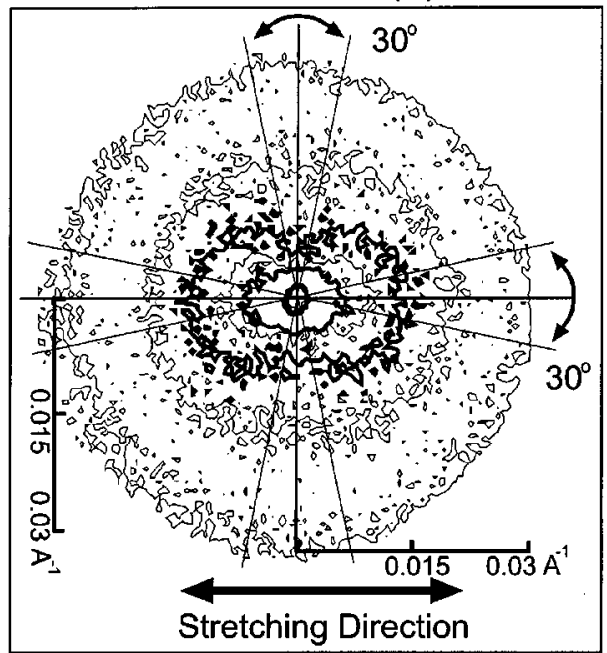

FIG. 3. Abnormal butterfly scattering pattern obtained for NIPA gel at (a) $28^{\circ} \mathrm{C}$, (b) $31^{\circ} \mathrm{C}$, and (c) $33^{\circ} \mathrm{C}$, with deformation ratio $\Lambda=1.5$. 

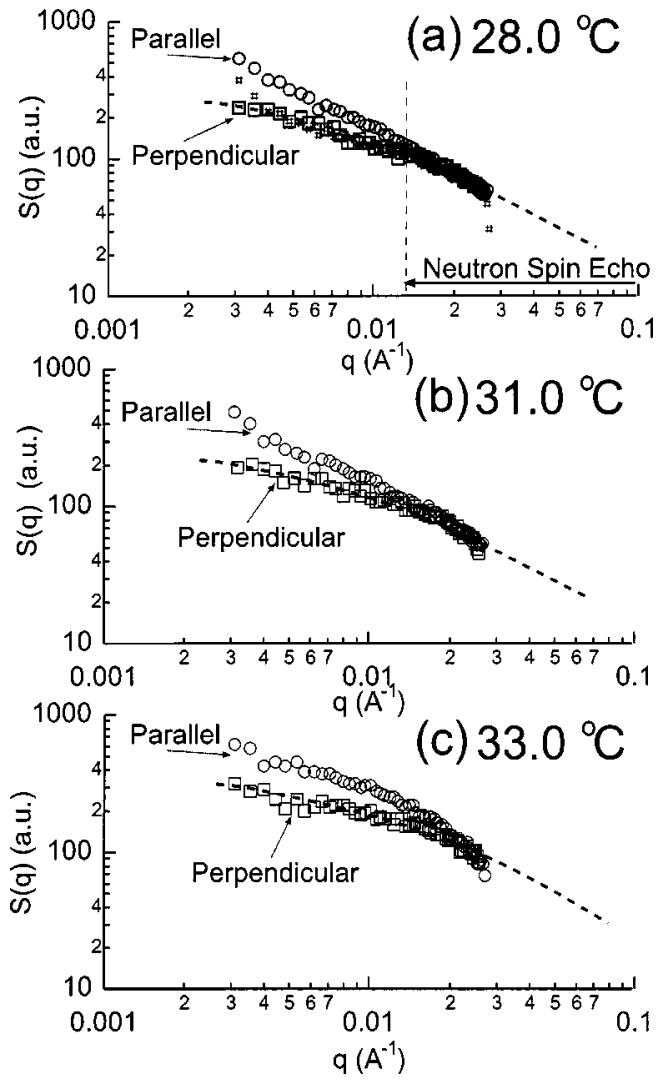

FIG. 4. Sector-averaged $q$-profiles of abnormal butterfly scattering for the NIPA gel, in directions parallel and perpendicular to stretching.

small within the error bars. The spectrometer IN15 at ILL, covering $t_{F}$ up to $180 \mathrm{~ns}$, plays an important role in determining slower decays for the low $q$, while for the high $q$, the shorter $t_{F}$ up to $22 \mathrm{~ns}$ by FRJ2-NSE is sufficient to observe complete decays. In this paper we discuss the slower decays for the NIPA gel related to the static inhomogeneity or $S_{\mathrm{st}}(q)$.

Figure 5(b) shows temperature changes of the decay curves both for the PNIPA gel and solution obtained at $q$ $=0.056 \mathrm{~A}^{-1}$ by IN15. The decay curves for the PNIPA solution does not change in the temperature region between 20.0 and $32.5^{\circ} \mathrm{C}$. At $20.0^{\circ} \mathrm{C}$, the decay curves from the PNIPA gel is almost identical with those from a PNIPA solution. The PNIPA gel shows significant retardation as the temperature increases closer to $T_{V}$. It should be recalled that the retardation for the PNIPA gel becomes less obvious, as $q$ increases, and finally at the highest $q\left(=0.16 \mathrm{~A}^{-1}\right)$, the two decay curves for the PNIPA gel and solution, respectively, become identical even for higher temperature. At $34.5^{\circ} \mathrm{C}$ in the collapsed phase, the decay curve shows an obvious plateau due to appearance of the PNIPA-rich microdomain, as observed by SANS in Fig. 2.

In order to determine local dynamics, we carefully examined the decay curves by NSE with the following methods; (i) first cumulant $\Omega$ (or the initial slope of decay curve), (ii) asymptotic decay behavior for long $t_{F}$, and (iii) Zimm scaling. ${ }^{25}$

For the PNIPA solution, we found that the first cumulant $\Omega$ changes according to $\sim q^{3}$ at higher $q\left(>0.05 \mathrm{~A}^{-1}\right)$, cor-
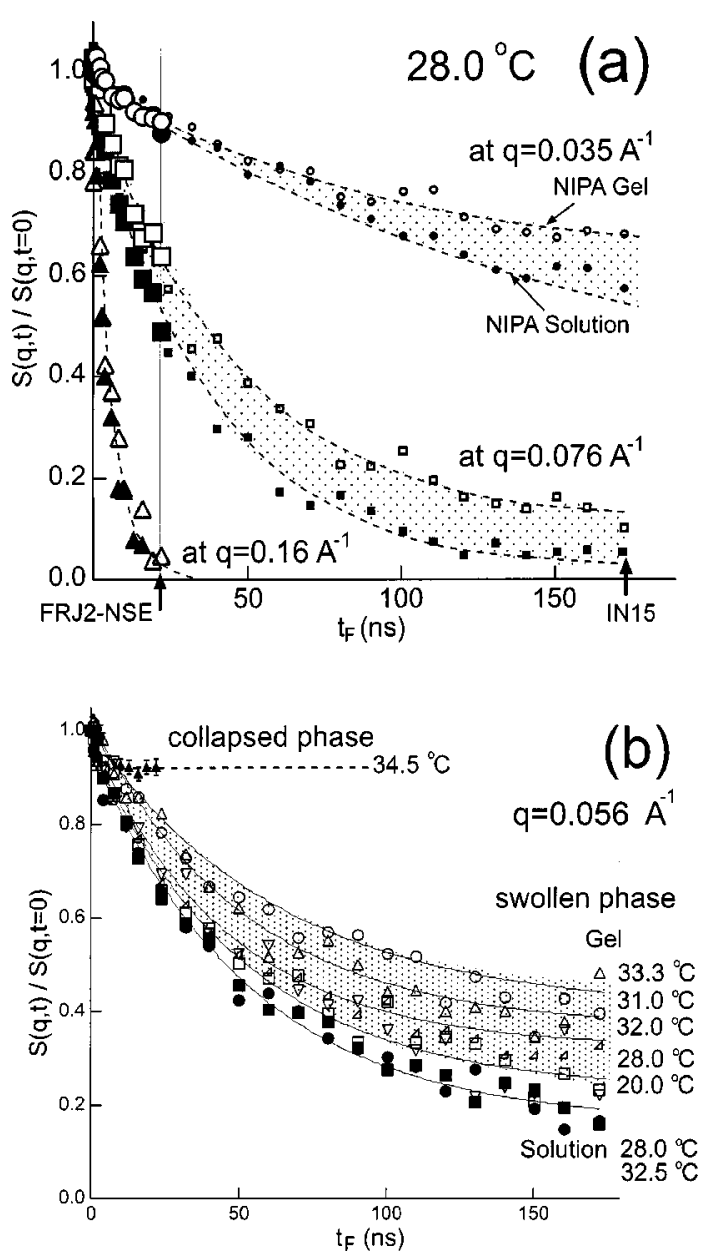

FIG. 5. (a) NSE decay curves $S(q, t) / S(q, t=0)$ at $q=0.035,0.076$, and $0.16 \AA^{-1}$, obtained for swollen NIPA gel (open symbols) and NIPA solution (filled symbols) at $28{ }^{\circ} \mathrm{C}$. The larger and smaller symbols indicate those obtained by FRJ2-NSE with $t_{F}=22 \mathrm{~ns}$ and by IN15 with $t_{F}=180 \mathrm{~ns}$. The shadows indicate the difference of two decay curves of the NIPA gel and solution (broken lines are guides for the eye). (b) Temperature changes of decay curves for NIPA gel and solution at $q=0.056 \AA^{-1}$, obtained by IN15.

responding to the internal modes of a Zimm single chain motion, whereas at lower $q\left(<0.05 \mathrm{~A}^{-1}\right)$, it changes according to $\sim q^{2}$, corresponding to the collective diffusion [see Fig. 6(a)]. Note that for a Zimm single chain motion, $\Omega$ is given as $\Omega=q^{3} T / \eta_{s}$ where $\eta_{s}$ is viscosity of solvent. ${ }^{25}$ The crossover behavior about the initial decay is same for the PNIPA gel, because the initial decays, observed in wide $q$, are identical both for the PNIPA gel and solution.

The long time behavior of the coherent dynamic structures is approximately given by

$$
\frac{S(q, t)}{S(q, t=0)} \sim \exp \left[-(\Omega t)^{\alpha}\right],
$$

where the exponent $\alpha$ is $2 / 3$ and 1 for the Zimm single chain motion and collective diffusion, respectively. Note that a double-logarithmic plot of the decay curve $(=\ln [-\ln [S(q, t) / S(q, t=0)]])$ provides $\alpha$ as a slope, which is shown in Fig. 6(b). For PNIPA solution, $\alpha$ changes from 2/3 to 1 at around $q=0.05 \mathrm{~A}^{-1}$.

The Zimm scaling plot scaled with $q^{3} t_{F}^{2 / 3}$ shows that the curves for high $q\left(>0.05 \mathrm{~A}^{-1}\right)$ fall onto the scaled master 

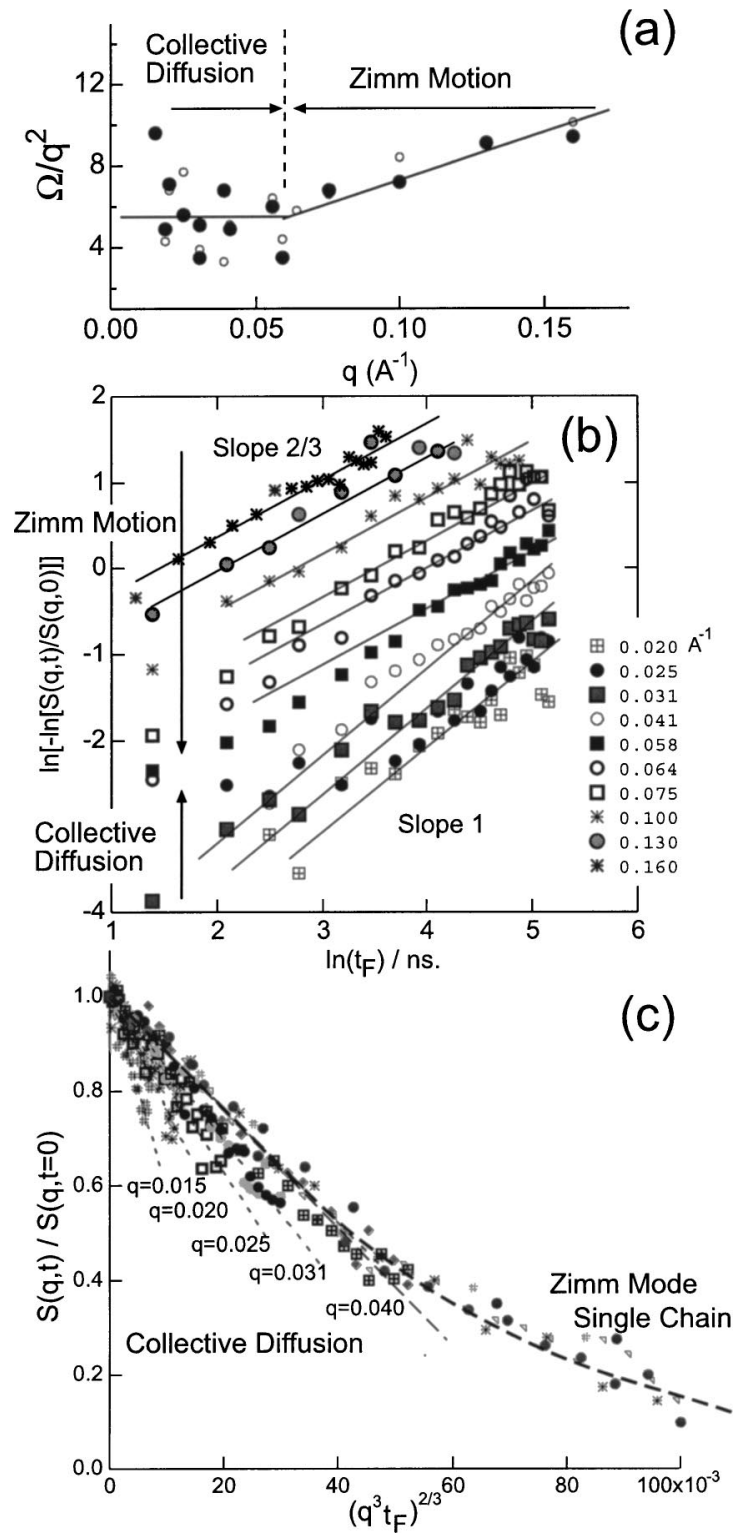

FIG. 6. Decay behaviors for NIPA solution examined by (a) first cumulant $\Omega$ where open and filled circles are obtained by FRJ2-NSE and IN15, respectively; (b) asymptotic decay behaviors at long $t_{F}$; and (c) Zimm scaling plot.

curve for the Zimm single chain motion, whereas at lower $q\left(<0.05 \mathrm{~A}^{-1}\right)$, the curves deviates from this master curve showing steeper slopes [see Fig. 6(c)].

From these observations, we now confirm for the PNIPA solution that for low $q\left(<0.05 \mathrm{~A}^{-1}\right)$, the intermediate scattering function $S(q, t) / S(q, t=0)$ decays according to the collective diffusion, whereas for high $q\left(>0.05 \mathrm{~A}^{-1}\right)$, it decays according to the Zimm single chain motion. For the PNIPA gel, this crossover of local dynamics is same except for the retardation of decays appearing at longer $t_{F}$.

Next, we attempt to explain the retardation found for a PNIPA gel. We attribute the apparent retardation to the static inhomogeneity coexisting with the thermal concentration fluctuations in the swollen gel. ${ }^{24}$ According to our scenario, $S(q)$ is given as an incoherent sum of $S_{\mathrm{st}}(q)$ and $S_{\mathrm{th}}(q)$

$$
S(q)=S_{\mathrm{st}}(q)+S_{\mathrm{th}}(q) .
$$

$S_{\mathrm{st}}(q)$ gives a nondecaying or elastic scattering component in the decay curve. On the basis of this scenario, we employ a scattering function with an elastic scattering ratio $P(q)$ as follows:

$$
S_{\mathrm{gel}}(q, t)=S_{\mathrm{gel}}(q, t=0)\{P(q)+[1-P(q)] f(t, q)\},
$$

where $f(q, t)$ is an universal relaxation function, describing a crossover from the collective behavior at low $q$ to the Zimm single chain behavior at high $q$. $\left[S_{\text {gel }}(q, t)\right.$ is the decay curve obtained for the PNIPA gel.] In order to proceed our analysis, we simply assumed that the universal function $f(q, t)$ can be replaced by the experimental curves obtained for the PNIPA solution; $f(t, q)=S_{\mathrm{sol}}(q, t) / S_{\mathrm{sol}}(q, t=0)$ where $S_{\text {sol }}(q, t) / S_{\text {sol }}(q, t=0)$ is the NSE decay curve for the PNIPA solution. This assumption is absolutely valid in high $q$ because two decay curves from the PNIPA gel and solution are same, obeying the Zimm single chain motion. Even for the lower $q\left(<0.035 \mathrm{~A}^{-1}\right)$, this assumption should be valid because we already found that the initial decays for the PNIPA gel are identical with the PNIPA solution. Therefore, by employing Eq. (3), we attempted to determine $P(q)$ experimentally at different $q$-positions.

$P(q)$ determined along our scenario increases as the temperature increases; at $q=0.015 \mathrm{~A}^{-1}, P(q)=0.24$ at $20.0^{\circ} \mathrm{C}, 0.35$ at $28.0^{\circ} \mathrm{C}$, and 0.55 at $33.3^{\circ} \mathrm{C}$. Then we evaluated $S_{\mathrm{st}}(q)$ according to $S_{\mathrm{st}}(q) \sim S_{\text {gel }}(q, t=0) P(q)$. Note that $S_{\text {gel }}(q, t=0)$ is equal to $S(q)$ by SANS. After subtraction of $S_{\text {st }}(q)$ from $S(q)$ observed by SANS, we obtained $S_{\text {th }}(q)$ originating from the thermal concentration fluctuations. Figure 7 shows $S(q)$ (open circles), $S_{\mathrm{st}}(q)$ (filled circles) and $S_{\mathrm{th}}(q)$ (open squares) thus determined at 28.0, 31.0 , and $33.0^{\circ} \mathrm{C}$. Here we notice that $S_{\mathrm{st}}(q)$ decreases rapidly at high $q$, according to $q^{-4}$. The strong upturn of $S(q)$ at low $q$ in Fig. 2 should relate to the larger-scale inhomogeneity studied by light scattering. ${ }^{13-15}$ The total contribution from static inhomogeneity should be smoothly connected between $S_{\text {st }}(q)$ determined here and the strong upturn, as indicated by lines in Fig. 7 .

Figure 8 shows temperature-changes of $S_{\mathrm{st}}(q=0.015)$, $S_{\mathrm{th}}(q=0.015)$ for the NIPA and forward scattering intensity $S(0)\left(=S_{\mathrm{st}}(0)+S_{\mathrm{th}}(0)\right)$ obtained for the PNIPA gel and solution. $S(0)$ were determined by Zimm plot analysis of $S(q)^{-1}$ versus $q^{2} .{ }^{12}$ The inverse forward scattering intensity $S(0)^{-1}$ changes according to $T^{-1}$ for the PNIPA gel and solution, as the temperature increases. It should be noted that $S(0)^{-1}$ for the NIPA solution changes more rapidly than that for the PNIPA gel; $S(0)^{-1} \sim 2.4 \times 10^{3} T^{-1}+$ const or $S(0)^{-1} \sim 5.9 \times 10^{3} T^{-1}+$ const for NIPA gel or solution, respectively. ${ }^{12}$

About $S_{\mathrm{st}}(q)$ and $S_{\text {th }}(q)$, on the other hand, we found as follows: (i) at $20^{\circ} \mathrm{C}, S_{\mathrm{th}}(q)$ dominantly occupies $S(q)$ and the static inhomogeneity is very small; (ii) as the temperature increases to $30.0^{\circ} \mathrm{C}, S_{\mathrm{st}}(q)$ increases according to $\sim T^{-1}$, while $S_{\text {th }}(q)$ increases more gradually according to $\sim T^{-1}$; and (iii) from near $30.0^{\circ} \mathrm{C}$, both $S_{\mathrm{st}}(q)$ and $S_{\mathrm{th}}(q)$ increase similarly and diverge at $T_{V}$. Thus we confirm that the temperature change of $S(q)$ for the PNIPA gel is also affected by $S_{\mathrm{st}}(q)$. Using static and dynamic light scattering, this steeper 

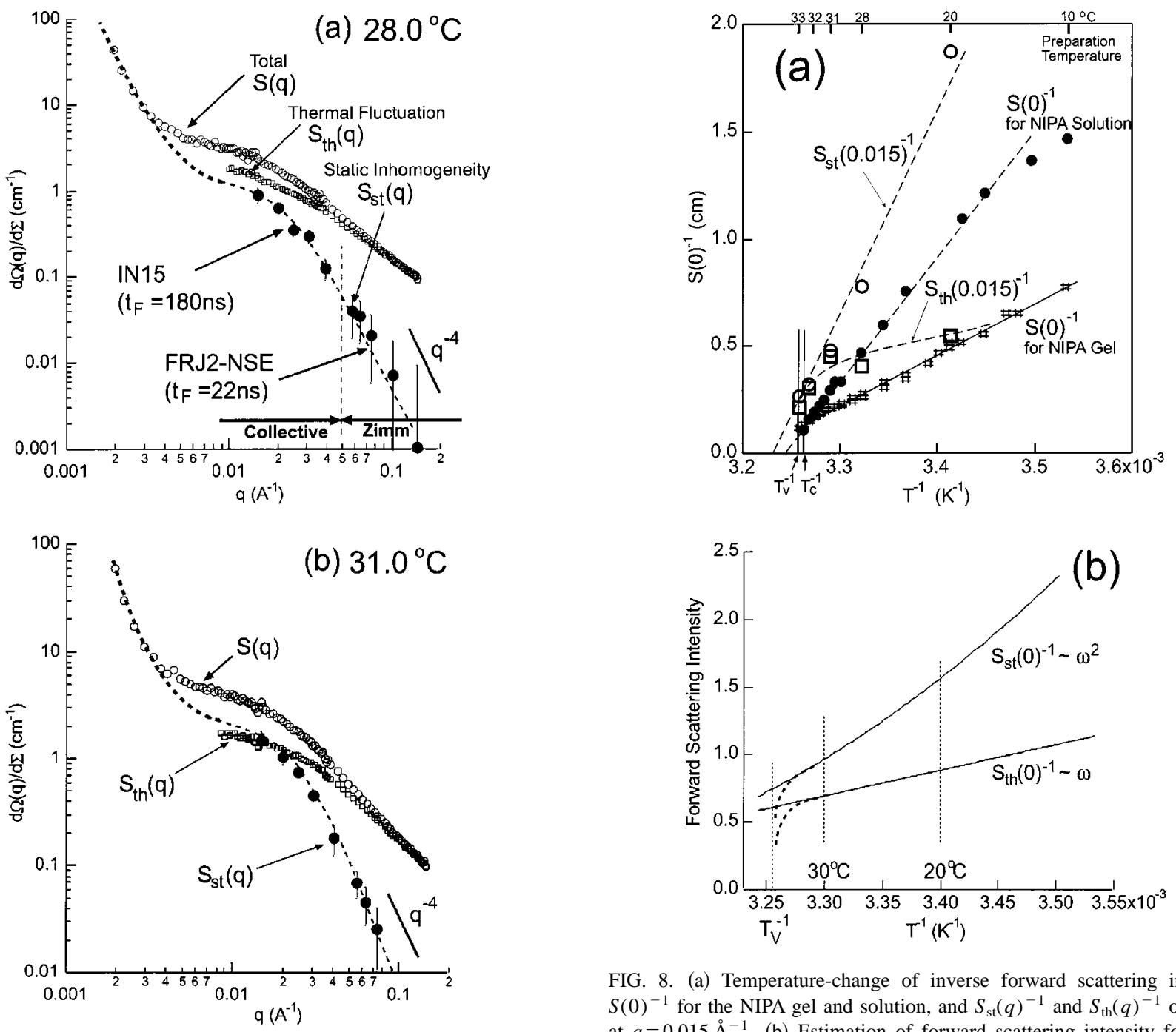

FIG. 8. (a) Temperature-change of inverse forward scattering intensity $S(0)^{-1}$ for the NIPA gel and solution, and $S_{\text {st }}(q)^{-1}$ and $S_{\text {th }}(q)^{-1}$ obtained at $q=0.015 \AA^{-1}$. (b) Estimation of forward scattering intensity for static and thermal scattering components, assuming to be proportional to the ex-

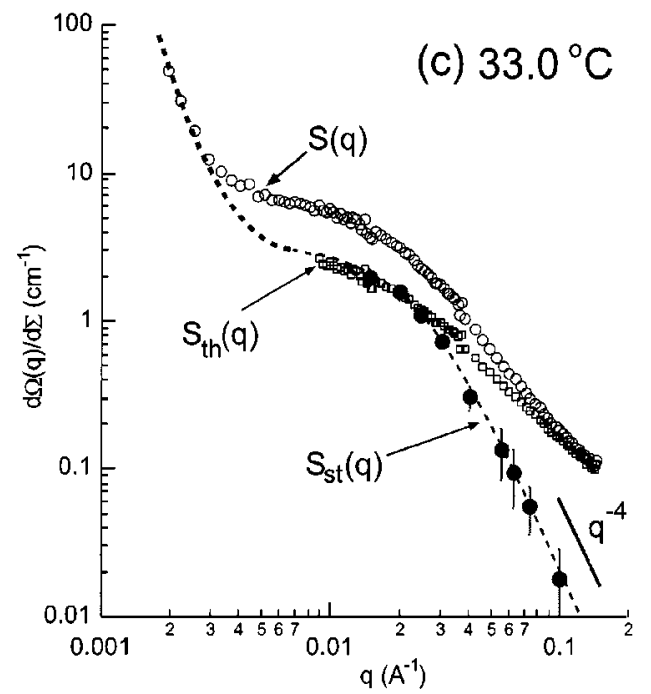
cluded volume parameter $\omega$ and $\omega^{2}$, respectively.

above $T_{V}$, which shows an asymptotic decay of $q^{-4}$ at high $q$, is attributed to the necklacelike microstructure where the NIPA-rich globules are connected by the swollen NIPA chains. ${ }^{12}$ At low $q$, the asymptotic decay of $S(q)$ becomes smaller. The crossover $q$ deviating from $q^{-4}$ corresponds to a characteristic size $R$ of globules in the necklacelike microstructure. $S_{\mathrm{st}}(q)$, shown by solid lines in Fig. 9, increases and seems to smoothly merge $S(q)$ from the necklacelike microstructure in the collapsed phase. This experimental finding suggests that the static inhomogeneity in the swollen phase closely relates to the PNIPA-rich necklacelike microdomain in the collapsed phase.

FIG. 7. Scattering curves, $S(q)$ (open circles) by SANS, $S_{\mathrm{st}}(q)$ (filled circles) and $S_{\text {th }}(q)$ (open squares) determined by NSE for the swollen NIPA gel at (a) $28{ }^{\circ} \mathrm{C}$, (b) $31{ }^{\circ} \mathrm{C}$, and (c) $33{ }^{\circ} \mathrm{C}$. The $q$-behaviors of $S_{\text {th }}(q)$ and $S_{\text {th }}(q)$ are similar to Lorentzian and squared Lorentzian shapes, respectively.

increase of $S_{\mathrm{st}}(q)$ has been reported in the continuum limit. ${ }^{26,27}$

In Fig. 9, we compare $S_{s t}(q)$ separated from $S(q)$ below $T_{V}$ and $S(q)$ obtained by SANS above $T_{V} . S(q)$ observed

\section{DISCUSSION}

The permanent crosslink in the chemical gel induces a strong coupling between stress and fluctuation. For the ideal gel, which is homogeneously crosslinked, the nonzero elastic modulus $\mu$ due to crosslinks is expected to suppress the thermal concentration fluctuations and therefore small-angle scattering. ${ }^{28}$ So far, there is no experimental observation of such suppression of small-angle scattering in the real gel. As 


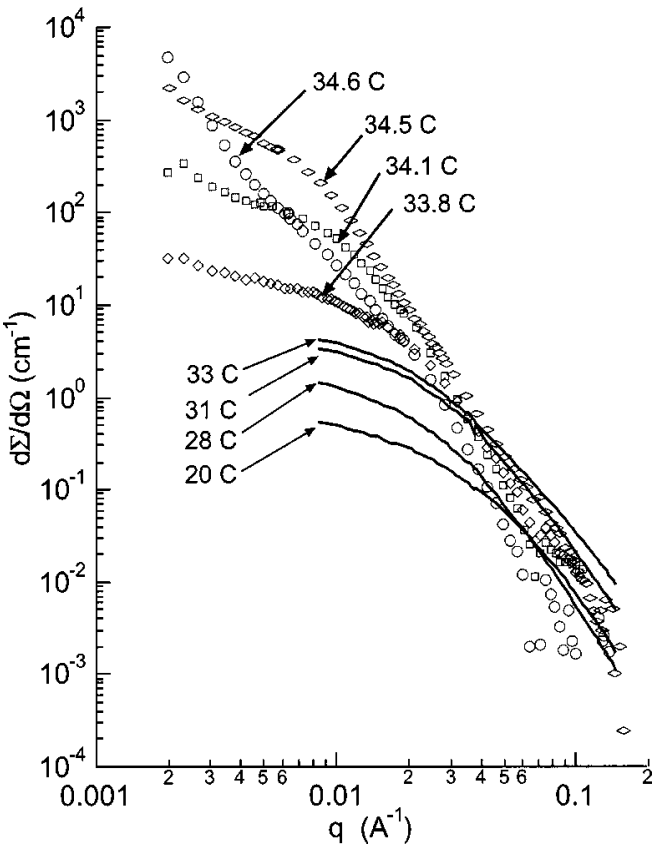

FIG. 9. Comparison between $S(q)$ by SANS in the collapsed phase above $T_{V}$ and $S_{\text {st }}(q)$ determined by NSE in the swollen phase above $T_{V}$.

related to this prediction, we have reported that the polymer mixture of polystyrene and poly (vinyl methylether), which has large difference in $T g$, shows anomalous suppression of small-angle scattering when the mixture is quenched in between two $T g$ 's. At the temperature between two $T g$ 's, the mixture behaves gel-like where the rheological relaxation becomes as slow as that of thermal concentration fluctuations. ${ }^{6}$ The slowly moving component of polystyrene behaves like a physical gel network dispersed in the fast moving component of poly (vinyl methylether). Here the rheological relaxation is determined by the slower component of polystyrene. In the dynamically asymmetric mixture, we have interpreted, the thermal concentration fluctuations are suppressed by a physical gel network. ${ }^{6}$

For the real chemical gel, which has a heterogeneous crosslink density, the scattering techniques often have revealed the opposite behavior: namely excess scattering as compared to the polymer solution. ${ }^{2}$ When the gel is deformed, the excess scattering is further developed as a socalled abnormal butterfly pattern. ${ }^{15,24,29-32}$ This is due to the static inhomogeneity, originating from the heterogeneous crosslink density.

Bastide $e t$ al. presented an intuitive understanding of the static inhomogeneity, i.e., frozen blob model. ${ }^{33}$ Permanent crosslinks prohibit a reconfiguration of polymer distribution when the gel is subjected to swelling or deswelling at conditions different from that of preparation. Upon swelling, the poorly crosslinked regions are more swollen, while the densely crosslinked regions are less swelling. The contrast in swelling is the origin of the static inhomogeneity in the real gel.

Concerning with the PNIPA gel, the phase boundaries of three different instabilities, as described in Introduction, exist near $T_{V}$. Below $T_{V}$, the PNIPA gel is well swollen with water. Especially at $10^{\circ} \mathrm{C}$ of gelation, water behaves as a good solvent so that we obtained a transparent gel. As the temperature increases toward the phase boundaries, the concentration fluctuations increase by heterogeneously changing the network configuration. Therefore, we observed increase of small-angle scattering by SANS. The combined method of SANS and NSE elucidated that the static inhomogeneity increases more dramatically as the temperature increases. Simultaneously, above $30^{\circ} \mathrm{C}$, the PNIPA gel starts to shrink gradually. These microscopic and macroscopic phenomena proceed according to the coupling effect between stress and fluctuations.

Concerning with the quantitative observations on $S_{\mathrm{st}}(q)$ and $S_{\mathrm{th}}(q)$, we summarize our findings:

We successfully decomposed $S(q)$ into $S_{\mathrm{st}}(q)$ and $S_{\mathrm{th}}(q)$, respectively. The $q$-behavior of $S_{\mathrm{st}}(q)$ or $S_{\text {th }}(q)$ is a squared Lorentzian or Lorentzian shape, respectively, showing an asymptotic decay of $q^{-4}$ or $q^{-2}$ (Fig. 7);

(ii) as the temperature increases toward $T_{V}, S_{\mathrm{st}}(q)$ increases in intensity more dramatically than $S_{\mathrm{th}}(q)$ (Fig. 8). This result agrees with the observations by static $^{26}$ and dynamic light scattering, ${ }^{27}$

(iii) the $q$-region, in which the abnormal butterfly scattering appears, corresponds to the low $q$-region, in which $S_{\mathrm{st}}(q)$ thus determined becomes dominant. The abnormal butterfly scattering is strongly related to the static inhomogeneity in the polymer gel (Figs. 3 and 7);

(iv) the static inhomogeneity in a swollen phase seems to grow up to the necklacelike microstructure in a collapsed phase (Fig. 9).

We further discuss our findings as compared to the recent theoretical approaches, ${ }^{17,18}$ which were independently developed to take into account the inherent structural heterogeneity in the real gel. The first model (Onuki No. 2) is developed in the framework of the elasticity for the continuum. ${ }^{18}$ This model postulates that $K_{\mathrm{os}}^{\mathrm{gel}}$ and $\mu$ vary randomly in space around the averages. According to the perturbation treatment, the structural nonuniformities play a role of the perturbation leading to the static component. In this model, $S(q)$ for the isotropically swollen gel, is given as the incoherent sum of a Lorentzian (Ornstein-Zernike) function from the thermal scattering and a squared Lorentzian function from the quenched static scattering (see Appendix A).

According to the Onuki No. 2 model, the ratio of $S_{\mathrm{th}}(0) / S_{\mathrm{st}}(0)$ is related to the elastic moduli as follows:

$$
\frac{S_{\mathrm{th}}(0)}{S_{\mathrm{st}}(0)}=\frac{K_{\mathrm{os}}^{\mathrm{gel}}+4 / 3 \mu}{\mu} \quad(=M / \mu) .
$$

Here we simply assumed that in Eq. (A4) the strength of inhomogeneity $P$ and swelling ratio $\left(\phi_{0} / \phi\right)$ are unity. From Fig. 8(a), we are able to estimate $S_{\text {th }}(q=0.015) /$ $S_{\mathrm{st}}(q=0.015)$, which is about 3.10 at $20.0^{\circ} \mathrm{C}$, while it decreases to about 1.86 at $28.0^{\circ} \mathrm{C}$. Finally above $31^{\circ} \mathrm{C}$, the ratio of $S_{\mathrm{th}}(q=0.015) / S_{\mathrm{st}}(q=0.015)$ becomes about 0.82 .

Geissler and his co-workers have discussed the longitudinal elastic modulus $M\left(=K_{\mathrm{os}}^{\mathrm{gel}}+\frac{4}{3} \mu\right)$ by using dynamic light 


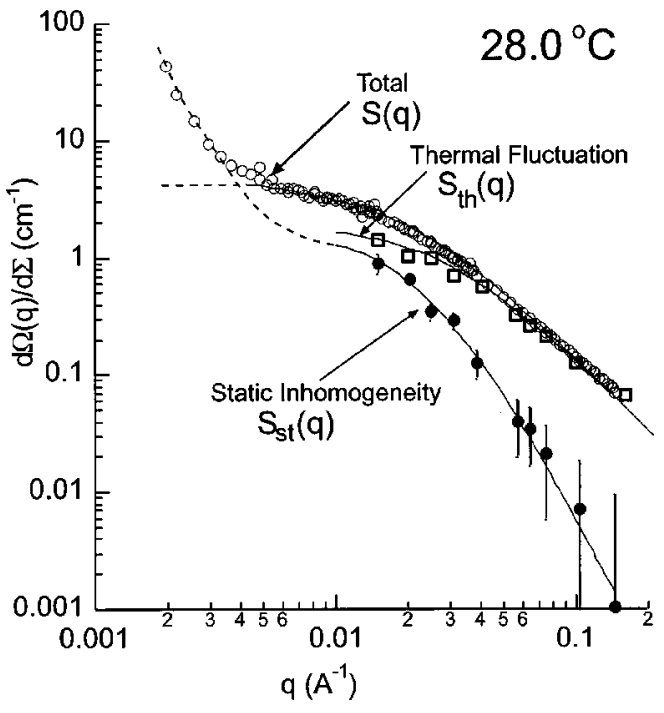

FIG. 10. $q$-profiles calculated by Panyukov-Rabin model (solid lines) bestfitted to $S(q)$ (open circles), $S_{\mathrm{st}}(q)$ (filled circles) and $S_{\mathrm{th}}(q)$ (open squares), obtained by SANS and NSE for the swollen NIPA gel at $28{ }^{\circ} \mathrm{C}$.

scattering and mechanical measurements. ${ }^{34}$ According to Ref. 34, the ratio of $M / \mu$ is given by the Poisson ratio $\sigma$ as follows:

$$
\frac{K_{\mathrm{os}}^{\mathrm{gel}}+4 / 3 \mu}{\mu}=\frac{2(1-\sigma)}{(1-2 \sigma)} .
$$

$\sigma$ for a single chain has been discussed depending on the solvent quality. ${ }^{35}$ Now we define $R_{\|}$and $R_{\perp}$ as the chain dimensions parallel and perpendicular to the compression direction. For the $\theta$-solvent state, the trajectories of a coil along three orthogonal directions are independent. Therefore, the Poisson ratio $\sigma\left(=-d R_{\perp} / d R_{\|}\right)$is zero in the case when we neglect a so-called log correction term discussed by Flory. For the good solvent state, the excluded volume effect is coupled among three orthogonal directions. Therefore, we obtain nonzero $\sigma$ varying around 0.25 ; Flory's description gives $\sigma=0.25$, while the scaling description by des Cloiseaux gives $\sigma=0.278$. On substituting into Eq. (5) the values of $\sigma=0$ or 0.25 for $\theta$ - or good solvent, we obtain

$\frac{K_{\mathrm{os}}^{\mathrm{gel}}+4 / 3 \mu}{\mu}=2$ for $\theta$-solvent or 3 for good solvent,

which quantitatively agrees well with our findings of $S_{\text {th }}(q$ $=0.015) / S_{\mathrm{st}}(q=0.015)$, ranging between 3.10 and 1.86 from $20.0^{\circ} \mathrm{C}$ to at $28.0^{\circ} \mathrm{C}$. Thus we confirm that from $20.0^{\circ} \mathrm{C}$ to $28.0^{\circ} \mathrm{C}$ the solvent quality changes from a good solvent to a $\theta$-solvent. The fact that above $30^{\circ} \mathrm{C}$, the ratio of $S_{\text {th }}(q=0.015) / S_{\text {st }}(q=0.015)$ is nearly equal to 1 implies the negative Poisson ratio; for $\sigma<0$ if we elongate the gel $\left(R_{\|}\right.$ $>0)$, the gel expands to perpendicular directions $\left(R_{\perp}>0\right)$. By mechanical measurements for the PNIPA gel, ${ }^{36}$ it was reported that the Poisson ratio becomes negative around $33^{\circ} \mathrm{C}$. Although the temperature found for the negative $\sigma$ is different, this result agrees with ours.

The second model is developed on the basis of the molecular structure in the gel [Panyukov-Rabin (PR) model], ${ }^{17}$ considering an instantaneous crosslinking of Gaussian chains. The chains are assumed to be "phantom"; knots or trapped entanglements are not considered. The excluded volume effect of segments is treated as a perturbation. According to the replica trick, this model calculate free energy for the gel with a heterogeneous crosslinking density, quenched during gelation. As described in Appendix B, $S(q)$ is again given as an incoherent sum of $S_{\mathrm{st}}(q)$ and $S_{\mathrm{th}}(q)$.

Figure 10 shows $S_{\mathrm{st}}(q)$ and $S_{\mathrm{th}}(q)$ calculated by the PR model by using Eqs. (B1) and (B2) with characteristic parameters of $N=650, \chi=0.52, \chi_{0}=0.34, \xi_{\mathrm{th}}=43.6 \mathrm{~A}, \quad \xi_{\mathrm{st}}$ $=11.8 \mathrm{~A}$, and $a=8.0 \mathrm{~A}$, where $N, \chi, \chi_{0}, \xi_{\mathrm{th}}, \xi_{\mathrm{st}}$, and $a$ are the degree of polymerization between crosslinks, a Flory interaction parameter at observation temperature, that at preparation, a correlation length for thermal fluctuation, that for the static fluctuation, a segmental length, respectively. Here we notice that the calculated curves of $S_{\mathrm{st}}(q)$ and $S_{\mathrm{th}}(q)$, which well reproduce our experiment of $q>0.015 \mathrm{~A}^{-1}$, behave as squared Lorentzian and Lorentzian functions for the static and thermal scattering components, respectively showing the asymptotic tail of $q^{-4}$ and $q^{-2}$.

In contrast to the excellent agreement in $q$ $>0.015 \mathrm{~A}^{-1}$, for low $q\left(<0.015 \mathrm{~A}^{-1}\right)$ we recognize a large discrepancy between the PR model and our experiments because of the strong upturn of small-angle scattering. This upturn behavior, similar to the Picot-Benoit effect for the polymer solutions, ${ }^{37}$ should be attributed to the static inhomogeneity of larger length scales. We denote that the degree of polymerization between crosslinks $N=650$, which was determined by the PR model, is much larger than the expected value about $N=50$, which can be calculated from our recipe of sample preparation (see Sec. II). It implies that the effective crosslink density is lower and densely crosslinked regions might exist with a distance $\Xi$ as illustrated in Fig. 1. This experimental finding strongly supports the hierarchical static inhomogeneity in the real gel. Thus, the total scattering function of the static inhomogeneity, covering a wide $q$-range from $\mu \mathrm{m}$ to $\mathrm{nm}$, must be given by a smooth line as indicated in Fig. 7 or 10. Actually focusing on the upturn scattering, the attempts to determine the static component were performed by the scattering techniques by many researchers in several steps: they found Gaussian function for heterogeneous density of crosslinks ${ }^{37}$ or for the heterogeneous concentration fluctuation, ${ }^{38}$ stretched exponential function $^{39}$ and very recently squared Lorentzian function. ${ }^{40}$ Although so many efforts have been made so far, the origin of the upturn scattering is still controversial depending on the types of gels studied.

According to these two models, the second term of the static inhomogeneity becomes dominant when $\Lambda$ (deformation ratio) or strength of inhomogeneity degree $P$ becomes larger. It is reported that this second term is the origin for "excess scattering" as compared to the polymer solution and for "abnormal butterfly scattering" under deformation. Our finding by neutron scattering methods is consistent with these models in senses that (i) the $q$-region where abnormal butterfly scattering appears corresponds to that where $S_{\mathrm{st}}(q)$ becomes dominant and (ii) $S_{\text {st }}(q)$ has asymptotic tail of $q^{-4}$. The models further predict that the thermal concentration fluctuations are suppressed along a deformation. However, 
we did not find such suppression of small-angle scattering along a deformation (Fig. 4). The PR model further predicts that in the $\theta$-solvent state, the normal butterfly appears, which is not observed so far and our future problem.

In Fig. 8, we obviously recognize that as the temperature increases, the static inhomogeneity increases more rapidly than the thermal component. In the PR or Onuki No. 2 model, $S_{\mathrm{st}}(q)$ and $S_{\mathrm{th}}(q)$ are coupled with each other through the excluded volume interaction parameter $\omega$ and the thermal correlator without the excluded volume interaction $g(q)$ (see Appendixes A and B). Note that in the Onuki No. 2 model, $\omega$ and $g(q)$ are included in $K_{\mathrm{os}}^{\mathrm{gel}}$. Thus the squared Lorentzian form for the static inhomogeneity induces a strong dependence of $\chi^{2}$. In order to confirm this situation, we estimated the excluded volume parameter $\omega$ and $\omega^{2}$. For this estimation, we employed $\chi(=\delta h-\delta s / T)$ as the enthalpic term $\Delta h=130 \pm 50\left(10^{-15} \mathrm{erg}\right)$ and the entropic term $\Delta s=-4.5$ $\pm 1.0\left(10^{-16} \mathrm{erg} \mathrm{K}^{-1}\right){ }^{41}$ In Fig. 8(b), we estimated interaction parameter $\omega$ and $\omega^{2}$ as a function of $1 / T$. As the temperature increases toward the phase boundaries, $\omega^{2}$ decreases more rapidly than $\omega$ as expected. The apparent change of $\omega^{2}$ seems linear as a function of $1 / T$. This is a reason why the static inhomogeneity increases more rapidly that the thermal scattering component. The similar argument has been done in Ref. 27. Near the phase boundaries, on the other hand, the linear change of $\omega$ and $\omega^{2}$ might be disturbed as schematically drawn by broken lines in Fig. 8(b). Unlikely for the van der Waals interacting system, $\chi$ for the NIPA-water mixture should be a strong function of temperature or of volume fraction $\phi^{35,42}$ near the phase boundaries, which is characteristic for the hydrophilic materials.

Above $T_{V}$, SANS shows a strong change of scattering intensity and its $q$-behavior and deviates from a Lorentzian shape showing an asymptotic $q$-behavior close to $q^{-4}$ for $q$ $>q_{0}(=1 / R)$ (see Fig. 2). We attribute the $q$-behavior to the necklacelike microstructure where a PNIPA-rich globulelike microstructure are connected by swollen chains. ${ }^{12}$ It has to be mentioned that this microstructure appears immediately after a temperature change above $T_{V}$ and stably exists at least more than a day. In Fig. 9, we found that $S_{\mathrm{st}}(q)$ determined in the swollen phase is similar to $S(q)$ from the necklacelike microstructures above $T_{V}$. This finding strongly suggests that the static inhomogeneity in the swollen state relates to the globules in the necklacelike microstructure. The size $R$ of the globule, determined as $R \cong 100 \mathrm{~A}$, might be determined by a force balance between entropic elasticity of swollen chains and the osmotic pressure. This balance plays a crucial role to pin the domain growth according to the stressfluctuation coupling. The pinning size should relate to the mesh size of the gel network and its inherent heterogeneity.

Related to the globule formation in the necklacelike microdomain, we refer to the Monte Carlo simulation performed for a single chain in a dilute solution. ${ }^{43}$ In Ref. 43, it is reported that the coil-globule transition proceeds through multi-steps; in an early stage, a chain locally collapses forming globules and then in a late stage, the globules aggregate and reach a complete collapsed state. For the polymer gel, the crosslink plays a crucial role to determine a critical nuclear size $R$ and to pin the aggregation process as predicted for a single chain.

\section{CONCLUSION}

By using a combined method of NSE and SANS, for the first time, we successfully decomposed $S(q)$ obtained by SANS into the static and thermal scattering components, respectively, $S_{\mathrm{st}}(q)$ and $S_{\mathrm{th}}(q)$ in the small-angle region of $0.015<q<0.16 \mathrm{~A}^{-1}$. The quantitative decomposition of $S(q)$ allows us to discuss the static inhomogeneity as follows: (i) as the temperature increases, $S_{\mathrm{st}}(q)$ of a squared Lorentzian shape increases more drastically than $S_{\mathrm{th}}(q)$ of a Lorentzian shape and (ii) $S_{\mathrm{st}}(q)$ is closely related to the abnormal butterfly scattering pattern appeared under stretching. These findings are quantitatively understood in the theoretical framework by Panyukov and Rabin or by Onuki, which are based on the stress-fluctuation coupling under coextitence of the inherent structural heterogeneity in the real gel. We further found that $S_{\mathrm{st}}(q)$ seems to grow to the necklacelike microstructure after a shallow quench in the collapsed phase.

\section{ACKNOWLEDGMENTS}

The author would like to thank Masahiko Annaka of Kyusyu Univ. for sample specimens. He acknowledges valuable discussions with Mistuhiro Shibayama of University of Tokyo and Tomohisa Norisuye of Kyoto Institute of Technology. He also wishes to acknowledge that this work was performed in collaboration with the neutron scattering group II of Jülich Research center, directed by Dieter Richter.

\section{APPENDIX A: THEORETICAL APPROACH I (ONUKI NO. 2)}

According to the Onuki (No. 2) model, the total scattering intensity is given as a sum of $S_{\mathrm{st}}(q)$ and $S_{\mathrm{th}}(q)$ given as follows:

$$
\begin{aligned}
& S_{\mathrm{th}}(\vec{q})=\frac{\phi^{2} \alpha}{\nu_{0}} \frac{1}{\epsilon_{\mathrm{el}}+J(\vec{q})+C(\vec{q}) q^{2}}, \\
& S_{\mathrm{st}}(\vec{q})=\frac{P \phi^{2} \alpha^{3}}{\nu_{0}}\left(\frac{J(\vec{q})-B \alpha^{-2}}{\epsilon_{\mathrm{el}}+J(\vec{q})+C(\vec{q}) q^{2}}\right)^{2},
\end{aligned}
$$

where $\epsilon_{\mathrm{el}}=K_{\mathrm{os}}^{\mathrm{gel}} / \mu+\frac{1}{3}$ and $\alpha=\left(\phi_{0} / \phi\right)^{1 / 3} \cdot V_{0}$ is the crosslink density. $J(q)$ is a function of the deformation ratio $\Lambda ; J(\vec{q})$ $=\left(\Lambda^{2}-\Lambda^{-1}\right) \cos \beta+\Lambda^{-1}$ where $\beta$ is an angle between the deformation and $q$-vector. The parameter $P$ denotes the strength of inhomogeneity: For larger $P(\cong 1)$, static inhomogeneity becomes dominant to show abnormal butterfly, while for small $P(\ll 1)$ it disappears. The physical meaning for the squared Lorentzian shape is intuitively understood as follows: the static fluctuation $\delta \phi_{\mathrm{st}}$ is proportional to the long range elastic deformation $\nabla u_{R}[\sim 1 /(1+q \Xi(1+q \Xi)]$ in reciprocal space. Thus the correlator $S_{\mathrm{st}}(q)$ becomes the squared Lorentzian shape. In case of isotropically swollen gel, where $J(q)=1$ and $B \alpha^{-1} \ll 1, S_{\text {st }}(q)$ becomes a simple squared Lorentzian form 


$$
S(q)=\frac{\phi^{2} T}{K_{\mathrm{os}}^{\mathrm{gel}}+4 / 3 \mu}\left[\frac{1}{1+\xi^{2} q^{2}}+\left(\frac{\Lambda^{2}}{\epsilon_{e l}+1}\right) \frac{p(q)}{\left(1+\xi^{2} q^{2}\right)^{2}}\right],
$$

where $\xi$ is the correlation length of thermal concentration fluctuations. In the long wave length limit of $q \rightarrow 0$, the ratio between $S_{\mathrm{th}}(q)$ and $S_{\mathrm{st}}(q)$ is written as

$$
\frac{S_{\mathrm{th}}(q)}{S_{\mathrm{st}}(q)}=P^{-1}\left(\phi_{0} / \phi\right)^{-2 / 3} \frac{K_{\mathrm{os}}^{\mathrm{gel}}+4 / 3 \mu}{\mu} .
$$

Note that this perturbation theory is not applicable near the phase boundary of $K_{\mathrm{os}}+\frac{4}{3} \mu \rightarrow 0$.

\section{APPENDIX B: THEORETICAL APPROACH II (PANYUKOV-RABIN)}

According to the Panyukov-Rabin (PR) model, $S_{\mathrm{st}}(q)$ and $S_{\mathrm{th}}(q)$ are obtained as follows:

$$
\begin{aligned}
S_{\mathrm{th}}(q)= & \frac{\phi \mathrm{N} g(q)}{1+\omega g(q)}, \\
S_{\mathrm{st}}(q)= & \frac{\phi N}{[1+\omega g(q)]^{2}\left(1+Q^{2}\right)^{2}} \\
& \times\left[6+\frac{9}{\omega_{0}-1+(1 / 2) Q^{2}\left[\phi_{0} / \phi\right]^{2 / 3} \phi_{0}^{-1 / 4}}\right],
\end{aligned}
$$

where $N$ and $Q$ are the degree of polymerization between crosslinks and the reduced wave number as $Q=\left[\frac{1}{6} \mathrm{Na}^{2} q\right]$. The subscript 0 in equations denotes the parameter at preparation temperature. The parameter $\omega$ denotes the excluded volume parameter for the mean-field approximation, including the Flory interaction parameter $\chi$

$$
\omega=(1-2 \chi+\phi) \phi N,
$$

$g(q)$ is the thermal correlator in the absence of the excluded volume effect.

$$
\begin{aligned}
S_{\mathrm{st}}(q)= & \frac{\phi N}{[1+\omega g(q)]^{2}\left(1+Q^{2}\right)^{2}} \\
& \times\left[6+\frac{9}{\omega_{0}-1+(1 / 2) Q^{2}\left(\phi_{0} / \phi\right)^{2 / 3} \phi_{0}^{-1 / 4}}\right], \\
g(q)= & \frac{1}{Q^{2} / 2+\left(4 Q^{2}\right)^{-1}+1}+\frac{2\left(\phi_{0} / \phi\right)^{2 / 3} \phi_{0}^{1 / 4}}{\left(1+Q^{2}\right)^{2}} .
\end{aligned}
$$

The static and thermal scattering components are coupled with each other through $\omega$ and $g(q)$. This situation is consistent with the Onuki No. 2 model, in which the excluded volume effect is included in the term of $K_{\mathrm{os}}^{\mathrm{gel}}$.

\footnotetext{
${ }^{1}$ M. Doi and A. Onuki, J. Phys. II 2, 1631 (1992).

${ }^{2}$ J. Bastide and S. J. Candau, in Physical Properties of Polymeric Gels, edited by J. P. Cohen Addad (Wiley, New York, 1996), pp. 143-308 and references cited therein.

${ }^{3}$ H. Tanaka, J. Phys.: Condens. Matter 12, R207 (2000).

${ }^{4}$ P.-G. de Gennes, Scaling Concepts in Polymer Physics (Cornell University Press, Ithaca, NY, 1979).
}

${ }^{5}$ N. Toyoda, M. Takenaka, S. Saito, and T. Hashimoto, Polymer 42, 9193 (2001).

${ }^{6}$ S. Koizumi, J. Appl. Crystallogr. 36, 381 (2003); S. Koizumi, J. Polym. Sci. Part B 42, 3148 (2004).

${ }^{7}$ A. Onuki, Phase Transition Dynamics (Cambridge University Press, Cambridge, 2002).

${ }^{8}$ T. Tanaka, L. O. Hocker, and G. B. Benedek, J. Chem. Phys. 59, 5151 (1973); T. Tanaka and D. J. Fillmore, ibid. 70, 1214 (1979).

${ }^{9}$ A. Onuki, Phys. Rev. A 38, 2192 (1988).

${ }^{10}$ A. Onuki and S. Puri, Phys. Rev. E 59, R1331 (1999).

${ }^{11}$ K. Sekimoto, N. Suematsu, and K. Kawasaki, Phys. Rev. A 39, 4912 (1989).

${ }^{12} \mathrm{~S}$. Koizumi et al. (in preparation)

${ }^{13}$ J. G. H. Joosten, J. L. McCarthy, and P. N. Pusey, Macromolecules 24, 6690 (1991); J. G. H. Joosten, A. Moussaid, and S. J. Candau, ibid. 27, 2102 (1994).

${ }^{14}$ M. Shibayama, T. Norisuye, and S. Nomura, Macromolecules 29, 8746 (1996).

${ }^{15}$ C. Rouf, J. Bastide, J. M. Pujol, F. Schosseler, and J. P. Munch, Phys. Rev. Lett. 73, 830 (1994).

${ }^{16}$ Neutron Spin Echo Lecture Notes in Physics 128, edited by F. Mezei (Springer-Verlag, Berlin, 1980).

${ }^{17}$ S. Panyukov and Y. Rabin, Macromolecules 29, 7960 (1996).

${ }^{18}$ A. Onuki, J. Phys. II 2, 45 (1992).

${ }^{19}$ T. Tanaka, Sci. Am. 244, 110 (1981).

${ }^{20}$ D. Schwahn, G. Meier, and T. Springer, J. Appl. Cryst. 24, 568 (1991).

${ }^{21}$ M. Monkenbusch, R. Schätzler, and D. Richter, Nucl. Instrum. Methods Phys. Res. A 399, 310 (1997).

${ }^{22}$ Guide to Neutron Research Facilities at the ILL (The Yellow Book) (Institut Laue-Langevin, Grenoble, 1997).

${ }^{23}$ S. Koizumi, M. Annaka, S. Borberly, and D. Schwahn, Physica B 276278, 367 (2000)

${ }^{24}$ S. Koizumi, M. Monkenbusch, D. Richter, D. Schwahn, and M. Annaka, J. Phys. Soc. Jpn. Suppl. A 70, 320 (2001); S. Koizumi, M. Monkenbusch, D. Richter, D. Schwahn, and M. Annaka, Appl. Phys. A: Mater. Sci. Process. 74, S402 (2002); S. Koizumi, D. Monkenbusch, D. Richter, D. Schwahn, and M. Annaka, J. Neutron Res. 10(3-4), 155 (2002).

${ }^{25}$ B. Ewen and D. Richter, Advances in Polymer Science, Vol. 134 (SpringerVerlag, Berlin, 1997).

${ }^{26}$ E. S. Matsuo, M. Orkisz, S. T. Sun, Y. Li, and T. Tanaka, Macromolecules 27, 6791 (1994).

${ }^{27}$ M. Shibayama, K. Kawakubo, and T. Norisuye, Macromolecules 31, 1608 (1998).

${ }^{28}$ A. Onuki, in Space Time Organization in Macromolecular Fluids, edited by F. Tanaka, T. Ohta, and M. Doi (Springer-Verlag, Berlin, 1989), p. 94.

${ }^{29}$ J. Bastide, L. Leibler, and J. Prost, Macromolecules 23, 1821 (1990).

${ }^{30}$ E. Mendes, Jr., P. Lindner, M. Buzier, F. Boué, and J. Bastide, Phys. Rev. Lett. 66, 1595 (1991).

${ }^{31}$ A. Ramzi, F. Zielinski, J. Bastide, and F. Boué, Macromolecules 28, 3570 (1995).

${ }^{32}$ E. Mendes, R. Oeser, C. Hayes, F. Boué, and J. Bastide, Macromolecules 29, 5574 (1996).

${ }^{33}$ J. Bastide and L. Leibler, Macromolecules 21, 2647 (1988).

${ }^{34}$ E. Geissler and A. M. Hecht, Macromolecules 13, 1276 (1980); 14, 185 (1981).

${ }^{35}$ S. Hirotsu, J. Chem. Phys. 94, 3949 (1991).

${ }^{36}$ H. Benoit and C. Picot, Pure Appl. Chem. 12, 545 (1966).

${ }^{37}$ E. Geissler, A. M. Hecht, F. Horkay, and M. Zrinyi, Macromolecules 21, 2594 (1988).

${ }^{38}$ S. Mallam, A. M. Hecht, E. Geissler, and E. Pruvost, J. Chem. Phys. 91, 6447 (1989)

${ }^{39}$ F. Horkay, A. M. Hecht, S. Mallam, E. Geissler, and A. R. Rennie, Macromolecules 24, 2896 (1991); F. Horkay, W. Burchard, A. M. Hecht, and E. Geissler, ibid. 26, 4203 (1993); E. Geissler, F. Horkay, and A. M. Hecht, J. Chem. Phys. 100, 8418 (1994).

${ }^{40}$ A. M. Hecht, F. Horkay, P. Schleger, and E. Geissler, Macromolecules 35, 8552 (2002).

${ }^{41}$ S. Hirotsu, J. Phys. Soc. Jpn. 56, 233 (1987).

${ }^{42}$ B. Erman and P. J. Flory, Macromolecules 19, 2342 (1986).

${ }^{43}$ Y. A. Kuznetsov, E. G. Timoshenko, and K. A. Dowson, J. Chem. Phys. 103, 4807 (1995) 